\title{
A New Low Threshold Platinized Epicardial Pacing Electrode: Comparative Evaluation in Immature Canines
}

\author{
PETER P. KARPAWICH, KENNETH B. STOKES, * JOHN R. HELLAND,* \\ CHERYL D. JUSTICE, and JACQELINE O. ROSKAMP
}

From the Section of Pediatric Cardiology, Department of Pediatrics, Children's Hospital of Michigan, Wayne State University, and *Medtronic, Inc., Minneapolis, MN

KARPAWICH, P.P., ET AL.: A new low threshold platinized epicardial pacing electrode: comparative evaluation in immature canines. A prospective comparison of pacing and sensing capabilities between the conventional Medtronic Model 4951 platinum-iridium epicardial pacing electrode and a new modified "platinized" version of the same electrode was performed in immature canines to determine if the new electrode design improves pacing in the immature myocardium. The conventional electrode was modified by electroplating platinum black particles onto the surface to increase the effective or true microscopic surface area, yet essentially maintain the same overall geometric electrode size. Both epicardial electrodes were inserted into the right ventricular myocardium with the lead pad sutured to the epicardium, and externalized to the scruff in five puppies (age 3 months). An additional left ventricular lead was implanted to permit chronic pacing following epicardially-induced atrioventricular block. Acute and chronic sensing and pacing capabilities of each externalized electrode were performed at implant and weekly up to 4 months. Histologic examination of each electrode implant site was performed at the end of the study period. At implant, both electrodes exhibited comparable values for sensed $R$ waves, lead impedances, and pacing thresholds. During the study, the platinized electrode exhibited lower pacing thresholds. Analysis of all postimplant data demonstrated this threshold difference to be significantly lower $(P<.01)$ for the platinized version. Lead impedance and sensing capabilities remained comparable between the two designs. Histologic study demonstrated less fibrotic infiltration at the platinized electrode site. This preliminary evaluation indicates that for the duration of the postimplant study period, the platinized epicardial electrode design was associated with significantly lower thresholds and less fibrosis as a function of time compared to the conventional smooth electrode surface design. The new platinized electrode limits exit block in the developing immature myocardium and permits safe pacing at lower pulse widths and voltages to increase battery life. (PACE, Vol. 11, August 1988)

platinization, cardiac pacing, epicardial electrode

\section{Introduction}

Electrode implant site fibrosis and exit block have been limiting factors to chronic epicardial

\footnotetext{
This study supported in part by grant 34-86 from the American Heart Association of Michigan, and grants from Children's Hospital of Michigan and Medtronic, Inc.

Address for reprints: Peter P. Karpawich, M.D., Section of Pediatric Cardiology, Children's Hospital of Michigan, 3901 Beaubien, Detroit, Michigan 42801.

Recieved April 10, 1987; revision January 20, 1987; revision February 17, 1987; accepted February 19, 1987.
}

and epimyocardial pacing in the young. Although recent application of the transvenous electrode implant approach to the pediatric age group has partially alleviated this problem, ${ }^{1,2}$ the need for efficient epicardial pacing persists. This need is most expressed in the very young and in those children with adverse cardiac or vascular anatomy which precludes the transvenous approach to cardiac pacing. Recent epicardial electrode designs which permit a limited depth of electrode penetration combined with secondary fixation characteristics are currently available and demonstrate improved pacing capability over pre- 
vious designs which caused extensive myocardial trauma. ${ }^{3,4}$ However, epicardial pacing continues to exhibit less effective chronic thresholds than the endocardial approach. The more recent introduction of steroid-eluting ${ }^{5,6}$ and platinum-plated ${ }^{7}$ endocardial pacing electrodes have demonstrated consistently better pacing and sensing thresholds than otherwise standard endocardial electrodes. Similar comparisons with epicardial electrodes are lacking.

This study represents the initial comparison of acute and chronic pacing and sensing capabilities between the standard polished platinum-iridium and new "platinized" versions of the Medtronic Model 4951 (Medtronic Inc., Minneapolis, MN, USA) "fish hook" epicardial pacing electrode in the immature, developing canine myocardium. The study was undertaken to determine if the new electrode surface design improves myocardial tissue-electrode reactivity and permits improved pacing capability in the young myocardium over the already established epicardial design.

\section{Materials and Methods}

\section{Preparation}

Five immature beagle puppies (age 3 months) were used in the study. All were instrumented following anesthesia with intravenous sodium pentobarbital and ventilated with room air following endotracheal intubation. Following a midline thoracotomy, the pericardium was incised to expose the right ventricle. In each animal, both a standard and new platinized version of the Medtronic Model 4951 epicardial pacing lead were sutured to the right ventricle, $1 \mathrm{~cm}$ apart. Electrode implant sites were alternated between animals to permit a nonbiased interpretation of myocardial reactivity. An additional left ventricular electrode, Medtronic Model 6917-35T, was also attached to later permit chronic pacing.

\section{Electrode Design}

The Medtronic Model 4951 epicardial electrode is a unipolar lead, insulated with polyure- thane (Pellethane 2363-80A). A distal 0.41 in. (11 $\mathrm{mm}) \times 0.75$ in. $(19 \mathrm{~mm})$ epicardial polyurethane pad provides stability for the electrode. A semicircular patch of Dacron fabric is attached to the proximal epicardial side of the pad to provide chronic fixation.

The electrode is formed from .020 diameter platinum alloy wire. The sharpened tip and distal barb provided a penetration and stabilizing configuration. The electrode surface area is nominally $10 \mathrm{~mm}{ }^{2}$ With the addition of the platinized finish, the true microscopic surface area is significantly increased, even though the macroscopic surface area remains at $10 \mathrm{~mm} .^{2}$

Platinization of the formed electrode is a three-step process. First, a two-stage acid boil and rinse process prepares the electrode material for electroplating. Then, platinum-black is deposited by electrochemical means using a D.C. voltage applied to the electrode while suspended in a platinizing solution. Finally, the electrode is rinsed in deionized water for several hours followed by an oven bake to dry.

The particles and their agglomerates are of such a small size that they absorb visible light resulting in a black appearance. This results in an extremely fine textured surface (Figures 1 and 2 ).

\section{Implant Technique}

Following fixation of both right ventricular electrodes, acute pacing, and sensing measurements were obtained using a Model 5311 pacing system analyzer (Medtronics, Inc.). Implant measurements for each electrode included voltage threshold at 0.5 milliseconds (ms), pulse width threshold at 2.5 and 5 volts (v), lead impedance, and sensed myocardial $R$ waves. A single left ventricular electrode was also attached and connected to an implanted pulse generator, Medtronic Model 8423 set in the VVI mode. After determination of optimal electrode position, each animal underwent transepicardial His bundle mapping and formation of complete atrioventricular block by direct epicardial formalin infiltration into the bundle of His. ${ }^{8}$ Chronic left ventricular pacing was then instituted.

The terminal ends of each right ventricular 


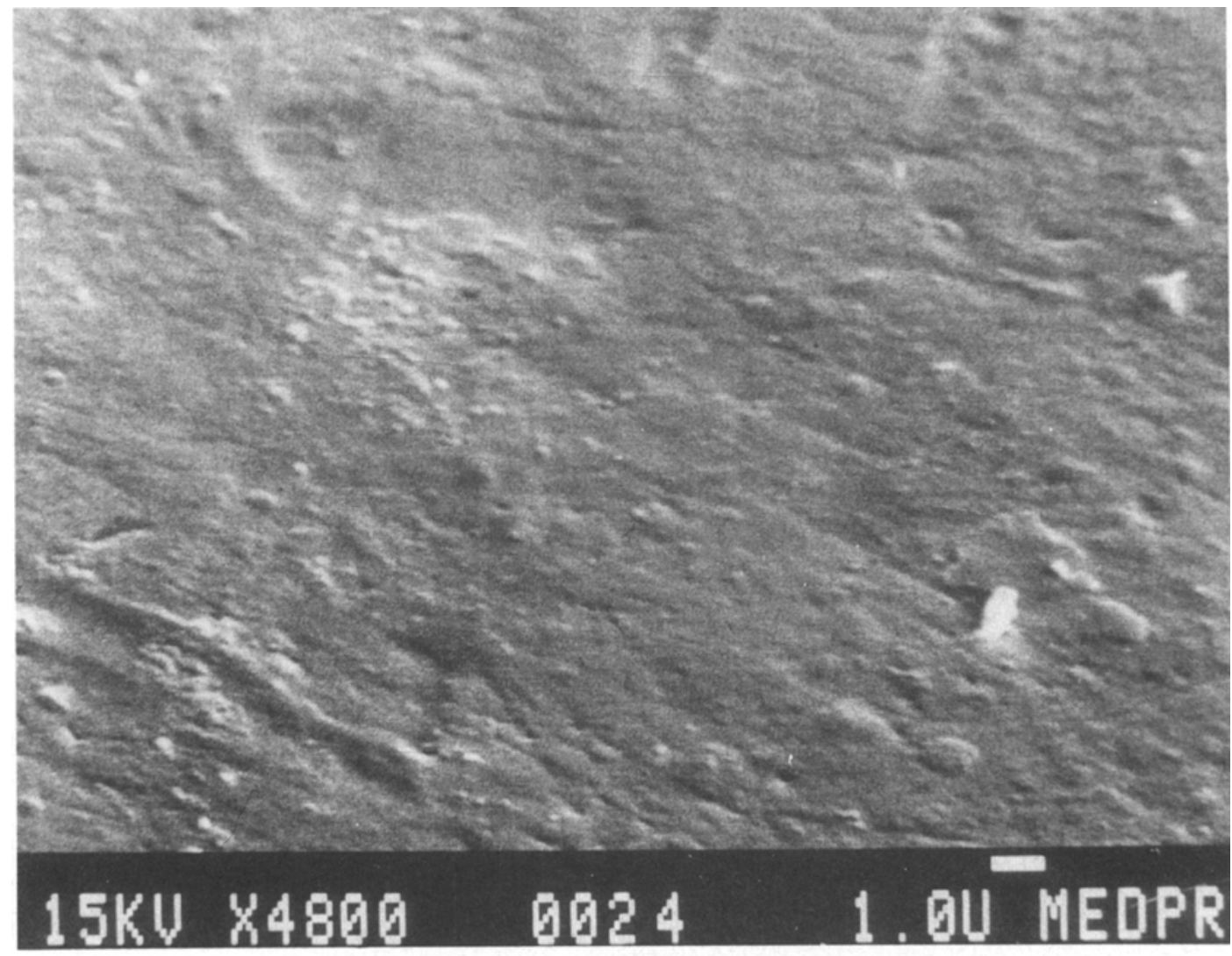

Figure 1. Electonmicroscopic appearance of conventional smooth platinum-iridium electrode surface. Calibration in lower right is 1 micron. Magnification is $4800 \times$.

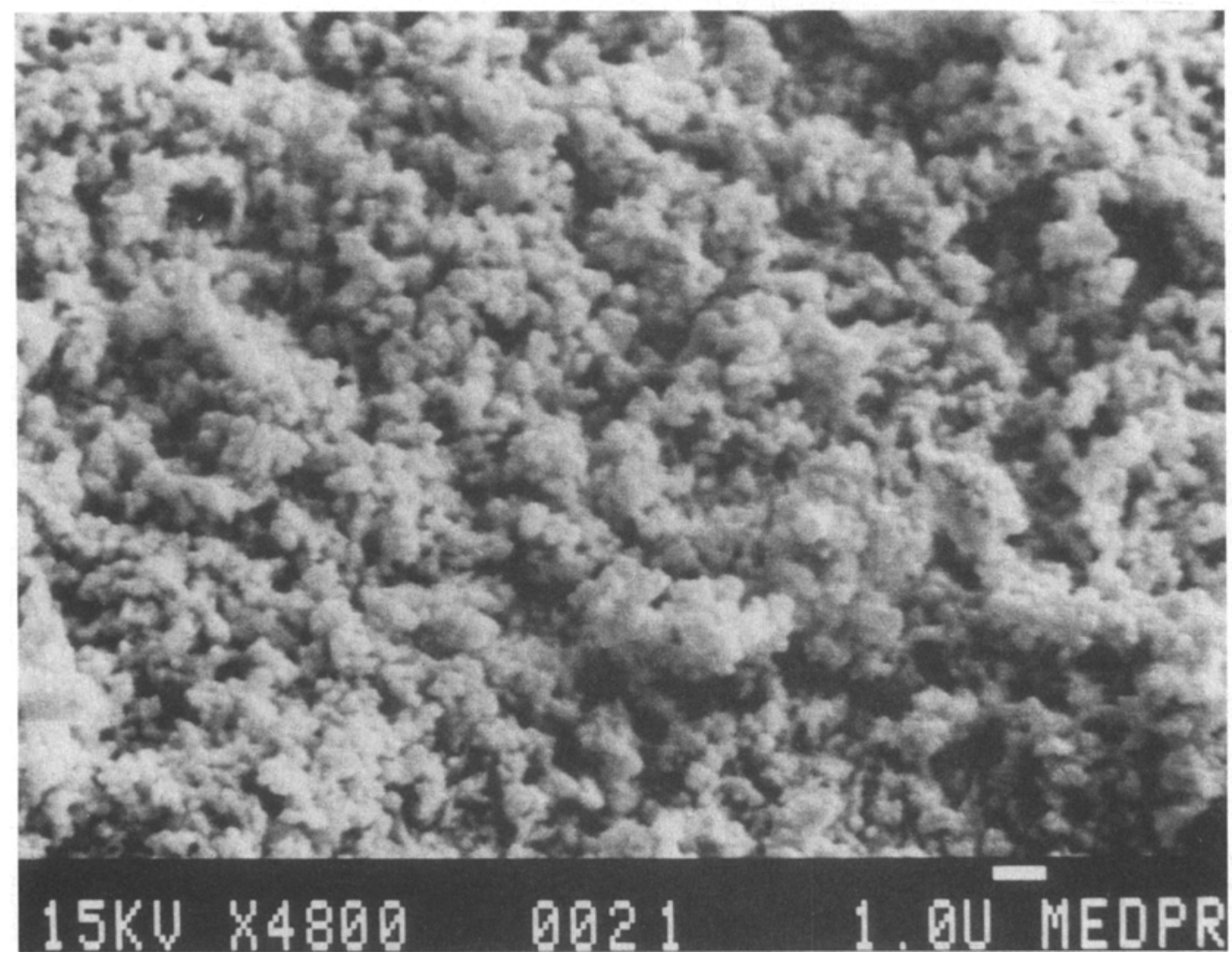

Figure 2. Comparative appearance of same platinum-iridium electrode after "platinization" illustrating fine microtextured surface on the order of 1 micronmeter or less. Magnification $4800 \times$. 
lead were then externalized to the scruff to later permit threshold and lead impedance testing. In this manner, the additional variable of cathode and anode electrodes was avoided in this small population preliminary study. The thoracotomy was closed in the usual fashion, and the animals allowed to recover. Weekly pacing and sensing characteristics of each externalized lead were determined in each puppy in the awake, nonsedated state using the pacing system analyzer. Measure- ments included lead impedance, voltage thresholds at $0.05,0.1,0.3,0.5,0.8$, and $2 \mathrm{~ms}$, as well as pulse width thresholds at $2.5,5$, and $10 \mathrm{v}$. Threshold was determined at each output setting by decremental programming the desired parameter until capture was lost, then incremental programming to capture. Threshold was defined as the lowest value prior to loss of capture.

At the termination of the study, each animal was sacrificed and the excised hearts fixed $(10 \%$

Table 1.

Electrode Pacing and Sensing Comparison at Implant* Mean Values

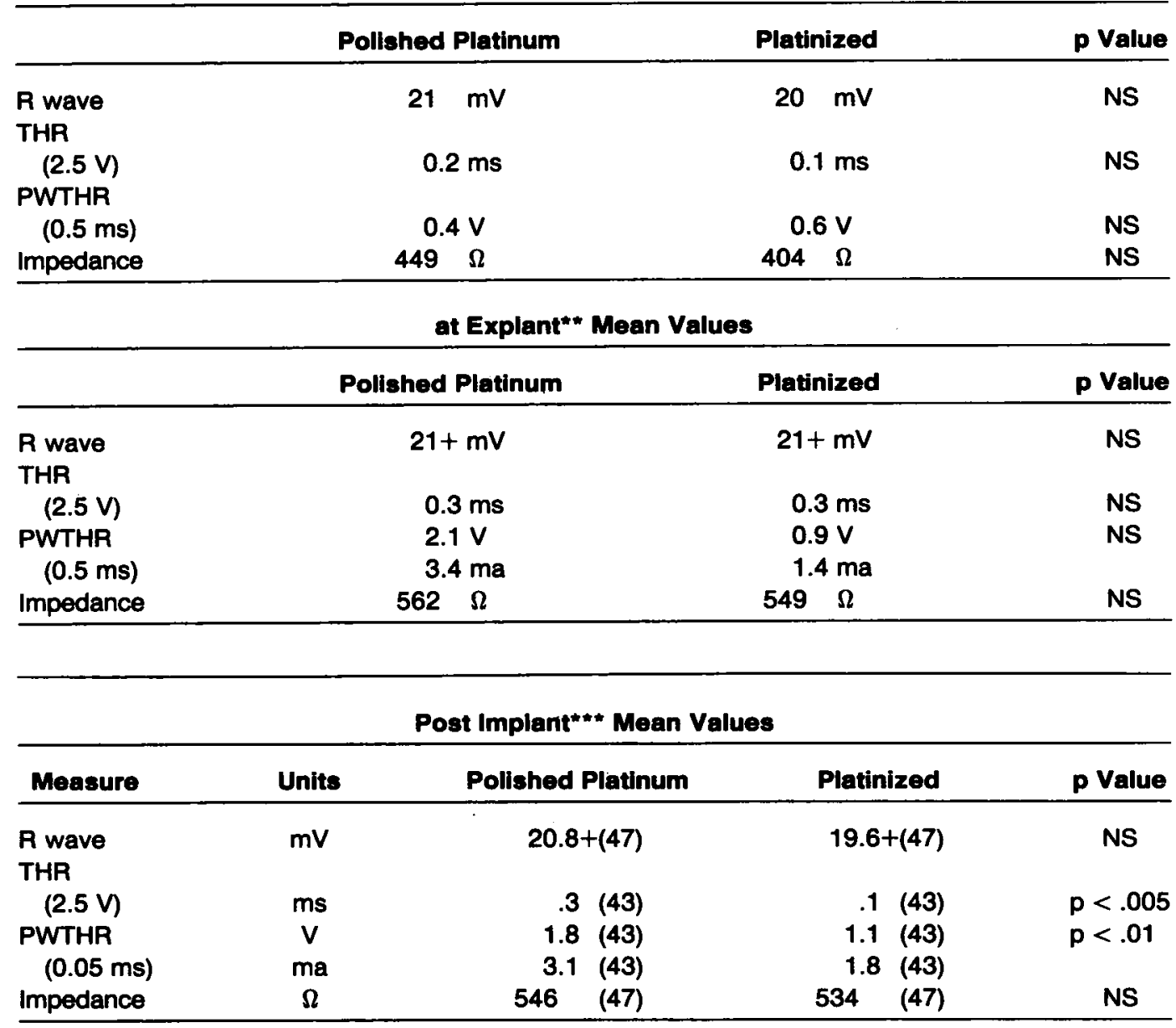

$* \mathrm{~N}=\mathbf{5}$ systems.

** $N=3$ systems. The two infected systems are excluded from in the data

*** Using three systems with repeated measurements over time. Two infected systems were excluded from the data. The number of measurements given in parenthesis.

THR: Threshold, PW: pulse width. 
formalin) for gross and microscopic examination of each electrode implant site using hematoxylin and eosin and trichrome staining methods. Specific attention was given to fibrotic changes in the myocardium.

\section{Stastical Analysis}

Implant data from each electrode version was compared using the paired t-test, with signifi- cance defined as a $P$ value of 0.05 . The small number of final electrodes tested precisely at explant, however, precluded any effective determination of significance using the paired t-test. Instead, consideration was taken of the random variation between the systems and within the systems for the duration of the postimplant period. All the postimplant data was therefore analyzed together using unbalanced random effects one-way analysis of variance. In this manner, each electrode

Table II.

Threshold (V/mA at $0.5 \mathrm{~ms}$ ) Versus Implant Time

\begin{tabular}{|c|c|c|c|c|c|c|c|c|c|c|c|}
\hline \multirow{2}{*}{\multicolumn{2}{|c|}{ Canines }} & \multicolumn{5}{|c|}{ Platinized Platinum } & \multicolumn{4}{|c|}{ Polished Platinum } & \multirow[b]{2}{*}{5} \\
\hline & & 1 & 2 & 3 & 4 & 5 & 1 & 2 & 3 & 4 & \\
\hline \multirow[t]{32}{*}{ Weeks } & 1 & 1.2 & 1.3 & .7 & 2.6 & 2.8 & 1.8 & 1.8 & 1.5 & 2.6 & 2.9 \\
\hline & & 2.5 & 2.5 & 1.7 & 4.3 & 5.4 & 3.5 & 2.8 & 3.7 & 4.3 & 5.5 \\
\hline & 2 & 1.1 & 1.2 & .7 & 2.1 & 1.8 & 1.4 & 1.7 & 1.5 & 2.3 & 2.3 \\
\hline & & 2.0 & 2.2 & 1.7 & 3.1 & 2.6 & 2.7 & 2.9 & 3.4 & 3.7 & 3.9 \\
\hline & 3 & .9 & 1.6 & .7 & 1.8 & 1.8 & 1.3 & 1.5 & 1.6 & 2.1 & 2.2 \\
\hline & & 1.7 & 3.0 & 1.7 & 2.7 & 2.9 & 2.5 & 2.7 & 3.8 & 3.1 & 3.5 \\
\hline & 4 & .8 & 1.8 & .6 & 1.8 & $\star$ & 1.4 & 1.6 & 2.2 & 2.2 & 2.0 \\
\hline & & 1.5 & 3.3 & 1.4 & 2.7 & & 2.6 & 2.8 & 4.9 & 3.0 & 3.5 \\
\hline & 5 & .8 & • & .5 & 1.9 & & 1.5 & * & 1.8 & 2.1 & $\star$ \\
\hline & & 1.5 & & 1.2 & 2.8 & & 2.7 & & 4.4 & 3.0 & \\
\hline & 6 & .7 & & .6 & 1.7 & & 1.5 & & 1.7 & 1.9 & \\
\hline & & 1.3 & & 1.5 & 2.6 & & 2.4 & & 4.3 & 2.9 & \\
\hline & 7 & .7 & & .6 & 1.5 & & 1.3 & & 1.7 & 1.7 & \\
\hline & & 1.3 & & 1.4 & 2.1 & & 2.3 & & 4.3 & 2.4 & \\
\hline & 8 & .6 & & .8 & 1.5 & & 1.5 & & 2.2 & 1.7 & \\
\hline & & 1.0 & & 1.3 & 2.1 & & 2.6 & & 3.5 & 2.4 & \\
\hline & 9 & .8 & & .8 & 1.4 & & 1.4 & & 2.7 & 1.8 & \\
\hline & & 1.4 & & 1.4 & 2.2 & & 2.5 & & 4.1 & 2.6 & \\
\hline & 10 & .8 & & .9 & 1.4 & & 1.4 & & 3.2 & 1.9 & \\
\hline & & 1.4 & & 1.5 & 2.3 & & 2.4 & & 5.1 & 2.9 & \\
\hline & 11 & .8 & & .7 & 1.5 & & 1.5 & & 3.2 & 1.9 & \\
\hline & & 1.4 & & 1.3 & 2.4 & & 2.6 & & 3.8 & 2.9 & \\
\hline & 12 & .8 & & & 1.3 & & 1.5 & & & 2.0 & \\
\hline & & 1.5 & & & 2.0 & & 2.8 & & & 2.8 & \\
\hline & 13 & .9 & & & 1.3 & & 1.4 & & & 1.9 & \\
\hline & & 1.8 & & & 2.0 & & 2.6 & & & 2.9 & \\
\hline & 14 & .8 & & & 1.3 & & 1.4 & & & 1.9 & \\
\hline & & 1.5 & & & 1.8 & & 2.7 & & & 2.6 & \\
\hline & 15 & .9 & & & 1.2 & & 1.4 & & & 2.0 & \\
\hline & & 1.6 & & & 1.5 & & 2.4 & & & 2.9 & \\
\hline & 16 & .8 & & & 1.3 & & 1.3 & & & 1.8 & \\
\hline & & 1.6 & & & 1.5 & & 2.3 & & & 2.1 & \\
\hline
\end{tabular}

* Infected 
version underwent up to 47 measurements to permit a more accurate determination of significance.

\section{Results}

Acute electrical implant and chronic explant data are listed in Table I. During the study, two systems became infected and are excluded from the explant data. At implant, there was no significant difference in any measured value between the two electrode versions. Table II lists voltage and milliampere (ma) thresholds obtained in each puppy at $0.5 \mathrm{~ms}$ pulse width, correlated over time. Mean values ( \pm standard error of the mean) for threshold are illustrated in Figure 3. The new platinized version exhibited consistently lower threshold values for the duration of the study.
Ventricular threshold strength-duration curves of pulse width and voltage 11 weeks following implant again exhibited consistently lower chronic values for the platinized electrode (Figure 4).

The small number of electrodes tested precisely at explant typically precluded any determination of significance between voltage and pulse width thresholds for the platinized compared to the polished platinum electrode versions. However, in comparison of all postimplant data obtained from each electrode version for the duration of the study, the platinized electrode exhibited a significant $(P<.01)$ improvement in threshold. Lead impedances and sensed myocardial $R$ waves remained comparable (Table I). Histologic evaluation demonstrated less reactive fibrotic infiltration associated with the platinized (Figure 5) compared to the standard electrode site (Figure 6).

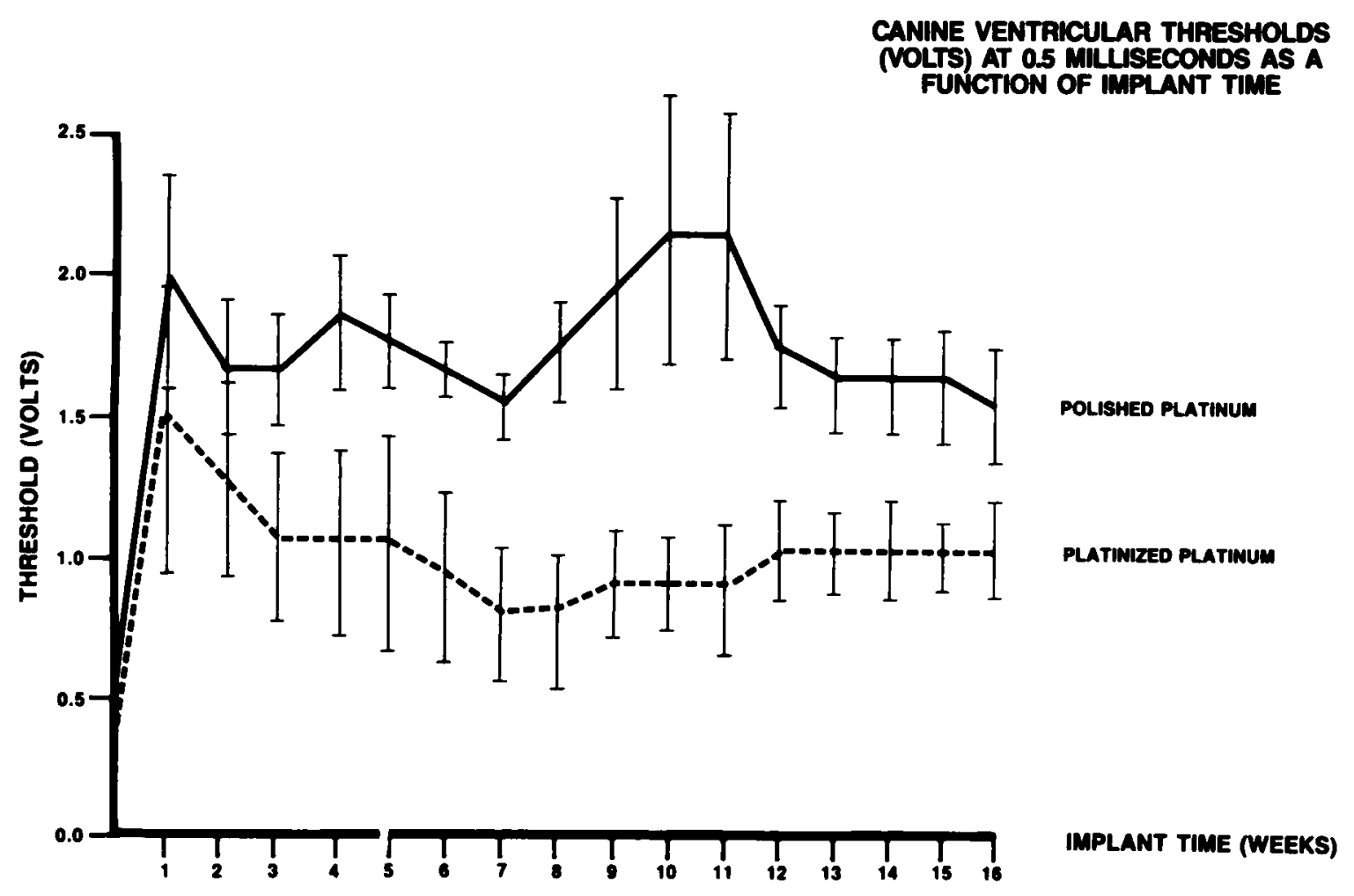

Figure 3. Serial ventricular thresholds at 0.5 milliseconds correlated over time illustrating comparative effect of platinization over conventional polished electrode surface. $N=3$ each lead. 


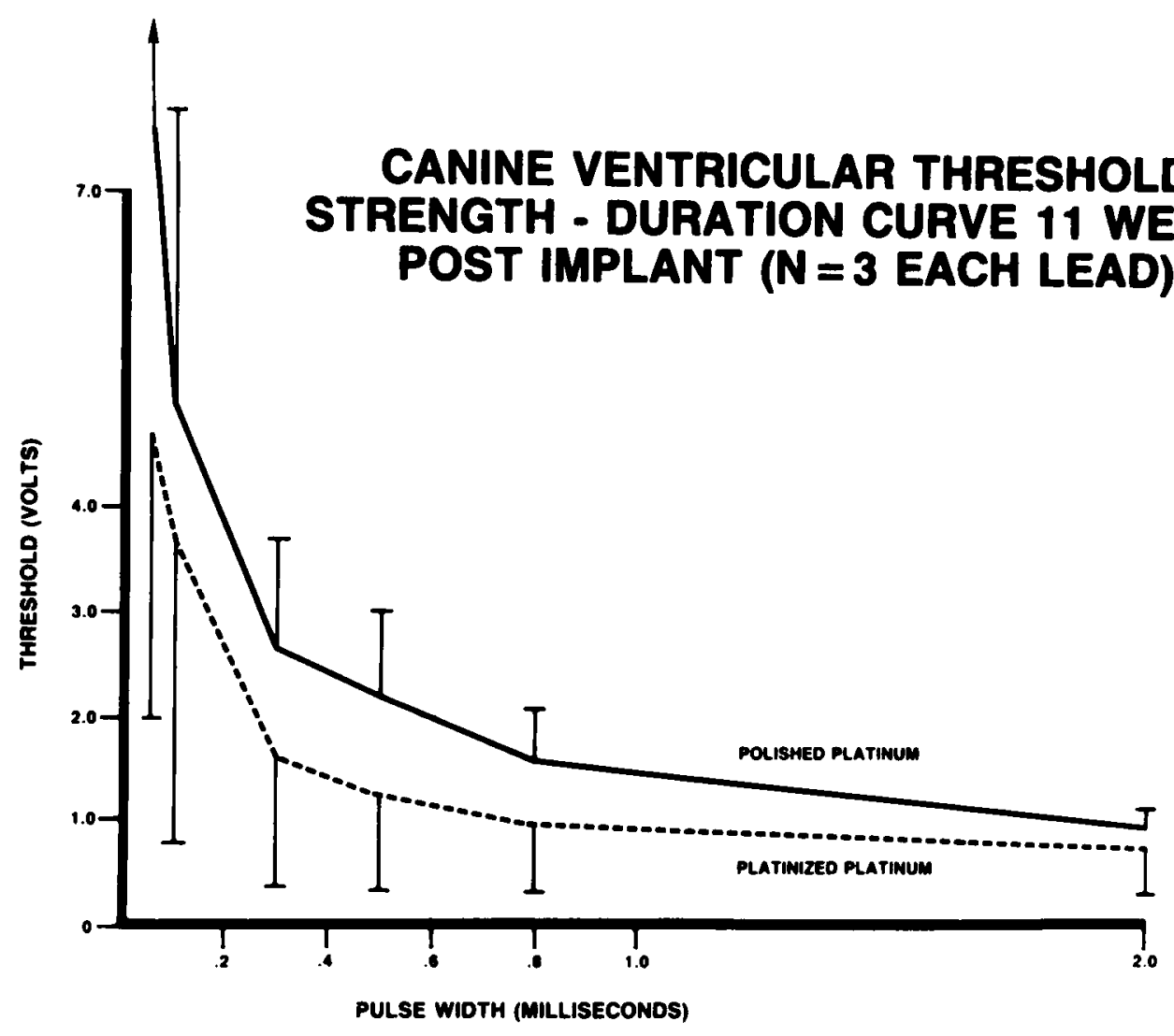

Figure 4. Mean strength-duration curves 11 weeks following implant.

\section{Discussion}

Although transvenous pacing systems are now applicable to some pediatric patients, the need for efficient epicardial pacing persists. This need is most apparent in the very young, in children with cardiac and vascular anomalies, which preclude a transvenous lead approach, and those who sustain conduction system damage during cardiac surgery. Unfortunately, the increased propensity for some children to develop electrode site fibrosis and exit block has limited effective chronic epicardial pacing by the requirement of higher voltage output and diminished pulse generator longevity. ${ }^{3,5}$ Although recent improvements in implant technique have helped to alleviate this problem, epicardial pacing in the imma- ture myocardium remains less efficient than comparable transvenous, endocardial pacing systems.

The recent advances seen with transvenous electrodes have been lacking for comparable epicardial systems. These include steroid-eluting electrode which permits chronic voltage thresholds approximately one-third of polished electrodes and one-half of similar porous electrodes without steroid. ${ }^{5.6}$ Another recent advance in transvenous lead technology involves the application of platinum black particles to the electrode surface. ${ }^{7,9}$ This latter advancement has also demonstrated efficient minimization of polarization losses. ${ }^{10}$ In experimental animal studies, chronic thresholds at $0.5 \mathrm{~ms}$ were approximately onethird of those of comparable polished platinum ring tip electrodes.' Similar findings for pulse 


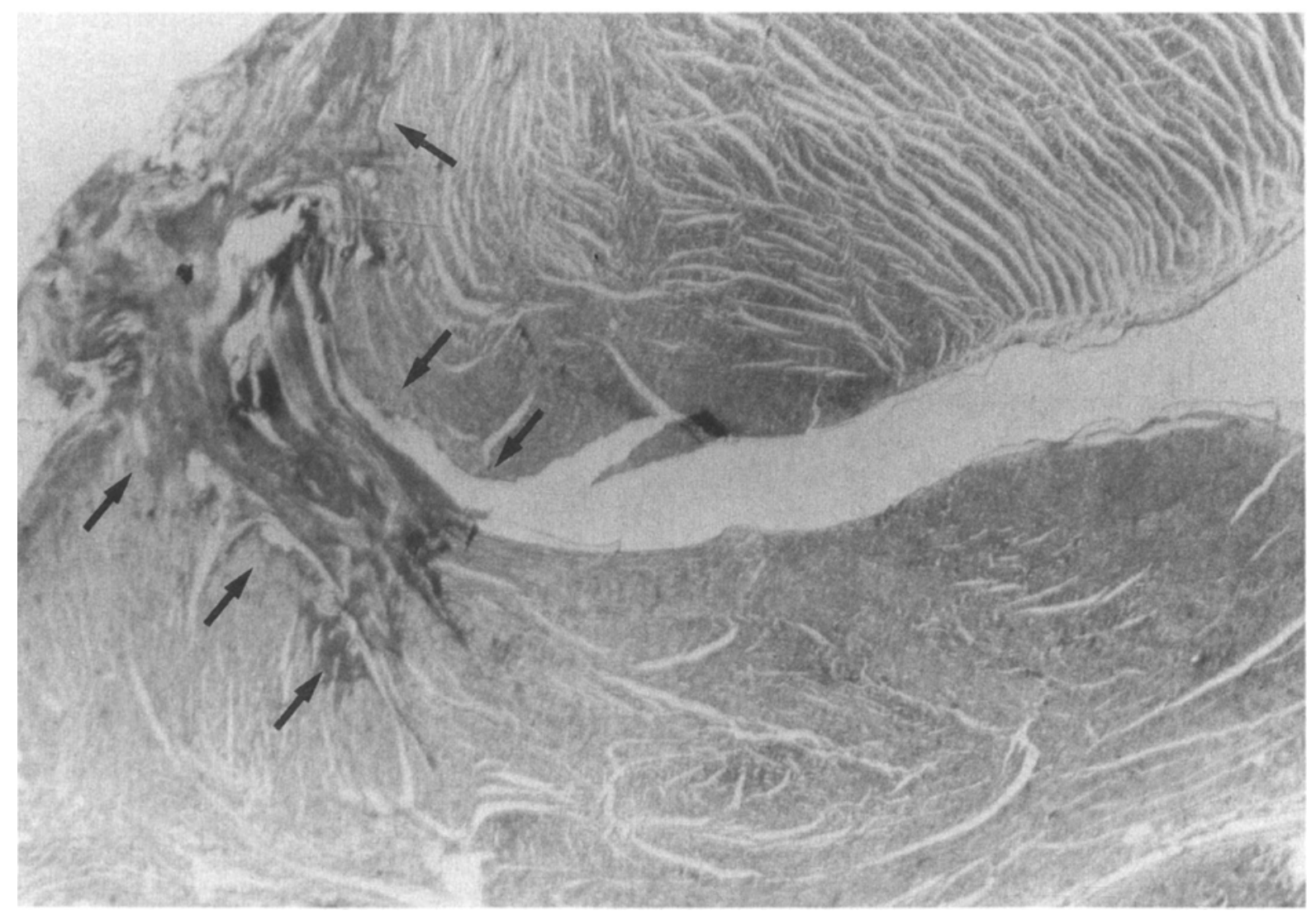

Figure 5. Histologic appearance of conventional smooth platinum-iridium electrode-epimyocardial tissue interface. Arrows outline extension of fibrotic infiltration which appears as darker stained material. Magnification $5 \times$.

width threshold at $5.4 \mathrm{v}$ have been reported for human clinical studies. ${ }^{9}$

This latter electrode technology has now been extended to epicardial pacing systems with comparable results. The ultra-fine surface texture of the platinized electrode appears to be beneficial for tissue acceptance of the foreign material. This concept is especially applicable to the epicardial implant surface where cellular reactivity can be extensive. Macrophage activation in response to the electrode and differentiation to multinucleated giant cells with release of lysosomal enzymes and oxidants results in cytotoxic cell damage, necrosis, and fibroblast proliferation. Collagen eventually encapsulates the electrode and replaces damaged myocardial tissue. If the electrode surface is modified to an ultra-small particulate surface, then the inflammatory response may be minimized. Macrophage proliferation and differentiation with ultimate collagen accumulation may be limited. This would result in a thinner fibrotic capsule and lower chronic thresholds. Studies are now underway to elucidate this hypothesis. ${ }^{11}$ In addition, the highly effective surface area minimizes polarization losses, permitting substantially more efficient transfer from electronic (in metal) to ionic (in tissue) conduction. ${ }^{9,12}$

These assumptions were supported in part during the histologic evaluation. The platinized 


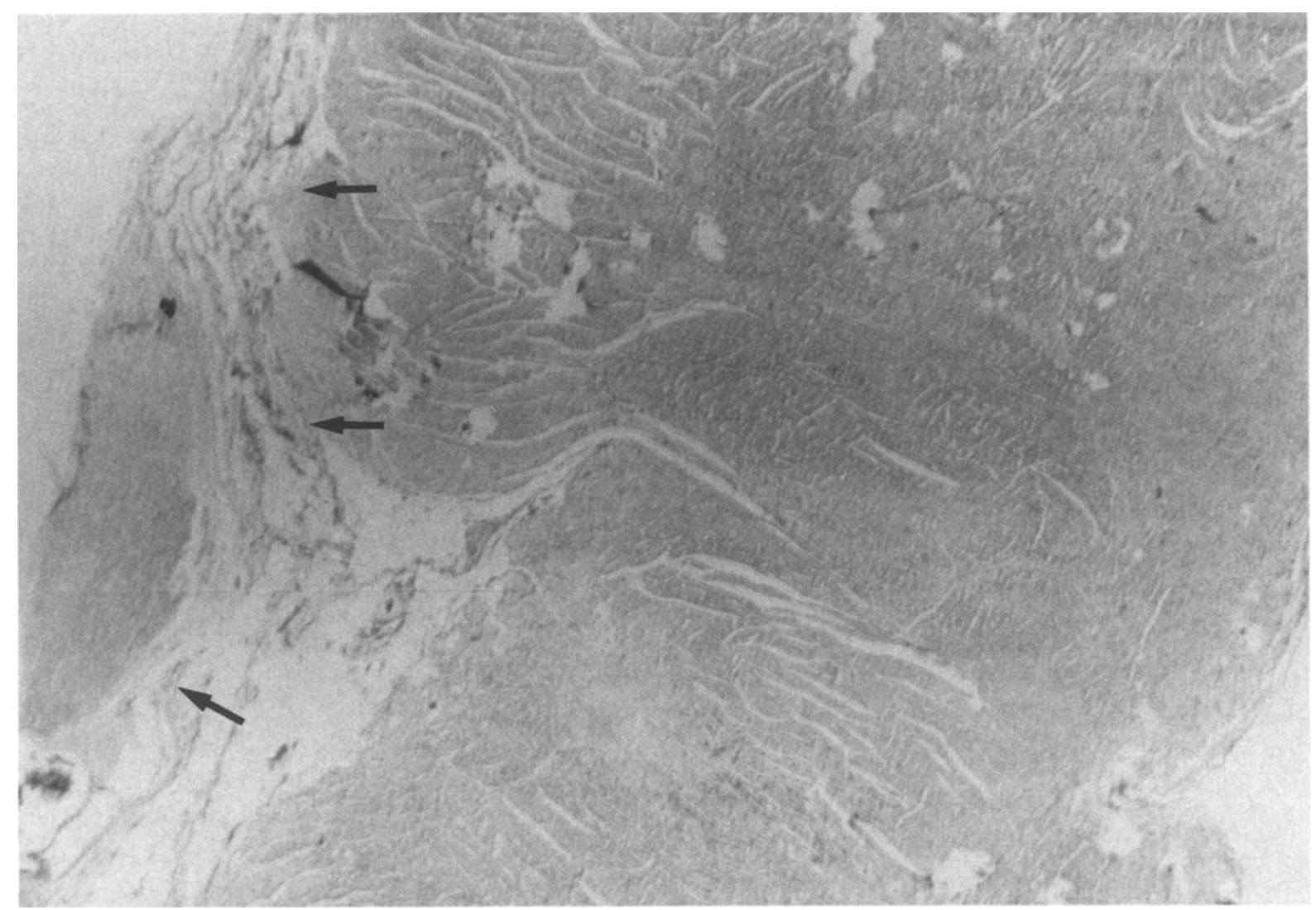

Figure 6. Comparative histologic appearance of new "platinized" electrode-epimyocardial tissue interface in small animal at comparable electrode postion in Figure 5. Arrows outline limited extent of fibrotic infiltration. Magnification $5 \times$.

electrode sites exhibited consistently less fibrotic infiltration than the smooth platinium-iridium sites.

Although this initial study was conducted on a small number of animals, these preliminary findings of improved pacing and sensing capabilities of platinized epicardial electrodes would indicate that epicardial pacing systems can be as efficient as current endocardial leads with wide application to the immature myocardium. The study was continued for 16 weeks after implant to assure chronic electrode stabilization. Due to this limited study duration, indefinite threshold stabilization can only be anticipated. However, the new platinized electrode was associated with significantly lower thresholds as a function of implant time for the duration of the study. Lower chronic strength-duration curves should allow safe pacing at lower pulse widths and voltages to increase battery life.

Acknowledgment: The authors wish to thank Janice McLarty for manuscript preparation and LeRoy Mattson for statistical analysis.

\section{References}

1. Gillette PC, Shannon C, Blair H, et al. Transvenous pacing in pediatric patients. Am Heart J 1983; 105:843.

2. Furman S, Young D. Cardiac pacing in children and adolescents. Am J Card 1977; 39:550.

3. Michalik RE, Williams WH, Zorn-Chelton S, et al. 
Experience with a new epimyocardial lead in children. PACE 1984 7:831.

4. Deleon SY, llbawi MN, Koster N, et al. Comparison of the sutureless and suture type epicardial electrodes in pediatric cardiac pacing. Ann Thorac Surg 1982; 33:273.

5. King D, Gillette PC, Shannon C, et al. Steroideluting endocardial pacing lead for treatment of exit block. Am Heart J 1983; 106:1438.

6. Kruse I. Long-term performance of endocardial leads with steroid-eluting electrodes. PACE 1986; 9:1217.

7. Bornzin GA, Stokes KB, Wiebusch WA. A low threshold, low polarization platinized endocardial electrode. (abstract) PACE 1983; 6:A-70.

8. Karpawich PP, Bharati S, Justice C, et al. Simultaneous transepicardlal his bundle mapping and ablation in immature canines: A precise technique for atrioventricular block. Cardiac Pacing and
Electrophysiology, Proceedings, VIIIth Symposium on Cardiac Pacing and Electrophysiology, 1987, pp 409-411.

9. Mohiuddin SM, Hee T, Butler ML, et al. Target-tip lead, clinical performance. (abstract) Clin Res 1984; 32:734A.

10. Tronstad A, Ohm OJ, Hoff PI, et al. Characterization of the polarization potentials from three modern endocardial pacemaker leads. In F Gomez (ed): Cardiac Pacing: Electrophysiology, Tachyarrhythmias, Mount Kisco, NY, Futura Publishing Company, Inc., 1985, p 1033.

11. Schoen FJ, Clagett GP, Hill JD, et al. The Bio-Capacity of Artificial Organs. Transactions, American Society of Artificial Implanted Organs, 33, No. 1987; 4:824-833.

12. Greatbatch $W$, Piersma B, Shannon F, et al. Polarization phenomena relating to physiological electrodes. Ann NY Acad Sci 1969; 167:722. 